\title{
Genome-wide analysis of NDR1/HIN1-like genes in pepper (Capsicum annuum L.) and functional characterization of CaNHL4 under biotic and abiotic stresses
}

\author{
Changyun Liu (1)', Haoran Peng ${ }^{2}$, Xinyu Li', Chaolong Liu', Xing Lv', Xuefeng Wei', Aihong Zou', Jian Zhang ', \\ Guangjin Fan', Guanhua Ma', Lisong $\mathrm{Ma}^{3}$ and Xianchao Sun ${ }^{1}$
}

\begin{abstract}
Plant NDR1/HIN1-like (NHL) genes play an important role in triggering plant defenses in response to biotic stresses. In this study, we performed a genome-wide identification of the NHL genes in pepper (Capsicum annuum L.) and characterized the functional roles of these CaNHL genes in response to abiotic stresses and infection by different pathogens. Phylogenetic analysis revealed that CaNHLs can be classified into five distinct subgroups, with each group containing generic and specific motifs. Regulatory element analysis showed that the majority of the promoter regions of the identified CaNHLs contain jasmonic acid (JA)-responsive and salicylic acid (SA)-responsive elements, and transcriptomic analysis revealed that CaNHL genes are expressed in all the examined tissues of pepper. The CaNHL1, CaNHL4, CaNHL6, CaNHL10, CaNHL11, and CaNHL12 genes were significantly upregulated under abiotic stress as well as in response to different pathogens, such as TMV, Phytophthora capsici and Pseudomonas syringae. In addition, we found that CaNHL4 localizes to the plasma membrane. CaNHL4-silenced pepper plants display significantly increased susceptibility to TMV, Phytophthora capsici and Pseudomonas syringae, exhibiting reduced expression of JA-related and SA-related genes and reduced ROS production. However, transient overexpression of CaNHL4 in pepper increases the expression of JArelated and SA-related genes, enhances the accumulation of ROS, and inhibits the infection of these three pathogens. Collectively, for the first time, we identified the NHL genes in pepper and demonstrated that CaNHL4 is involved in the production of ROS and that it also regulates the expression of JA-related and SA-related genes in response to different pathogens, suggesting that members of the CaNHL family play an essential role in the disease resistance of pepper.
\end{abstract}

\section{Introduction}

In nature, plants face constant attacks from insects and a diverse array of pathogens, such as bacteria, fungi, and viruses. However, plants have evolved sophisticated defense mechanisms to resist infection by these attackers. Plants have developed a two-tiered pathogen-recognition

\footnotetext{
Correspondence: Lisong Ma (Lisong.ma@anu.edu.au) or

Xianchao Sun (sunxianchao@163.com)

'Laboratory of plant immunity and ecological control of plant disease, College of Plant Protection, Southwest University, 400716 Chongqing, China

${ }^{2}$ Department of Botany and Plant Biology, Section of Biology, Faculty of Science, University of Geneva, 1211 Geneva 4, Switzerland

Full list of author information is available at the end of the article
}

system in which they employ either membrane-localized pattern-recognition receptor proteins or cytosolic nucleotide-binding leucine-rich repeat (NB-LRR) receptor proteins to sense extracellular or intracellular immunogenic compounds secreted by pathogens. Once perceived, these compounds trigger plant defense responses against various pathogens ${ }^{1-3}$. Pathogen resistance mediated by resistance proteins, especially NB-LRRs, is usually associated with the hypersensitive response (HR), which is characterized by rapid programmed cell death $(\mathrm{PCD})$ to limit the spread of the pathogen from the infection site ${ }^{4,5}$. The accumulation of reactive oxygen species (ROS) and 
nitric oxide (NO) at the pathogen infection site is considered important to initiate the HR, and other features, such as cell wall fortification, transcriptional reprogramming, and ion flux, are often observed ${ }^{5,6}$. Activation of the HR often induces systemic acquired resistance, which often relies on the salicylic acid (SA)-signaling pathway and the production of pathogenesis-related proteins ${ }^{5}$.

NDR1/HIN1-like (NHL) genes include Harpin-induced gene 1 (HIN1) and Nonrace-specific disease resistance gene 1 (NDR1) of Arabidopsis thaliana ${ }^{7}$. HIN1 is induced by harpin protein and plays an important role in various plant defense responses, growth, development, and resistance to abiotic stresses ${ }^{8}$. NDR1 has been cloned from $A$. thaliana and has been found to function in several plant disease resistance responses ${ }^{7}$. Most NHL proteins contain a conserved late embryogenesis abundant (LEA) domain", which belongs to a protein family whose members are largely related to osmotic regulation in different organisms. Previous studies have shown that the genes encoding proteins belonging to this protein family are highly expressed during the late embryonic development of seeds as well as under environmental stresses, such as drought and low temperature ${ }^{10}$.

It has been documented that members of the NHL family play an important role in plant disease resistance ${ }^{9}$. Maldonado et al. found that overexpression of GmNHL1 and GmNHL8 in A. thaliana plants enhanced resistance to Heterodera glycines by activating the jasmonic acid (JA) and ethylene (ET) pathways, suggesting that NHL1 and NHL8 contribute to the plant defense response against pathogens ${ }^{11}$. Chen et al. reported that overexpression of StPOTHR1, a member of the NHL gene family, enhances resistance against Phytophthora infestans by restricting rapid pathogen proliferation ${ }^{12}$. Overexpression of VvNHL1 (NHL 1) of Vitis vinifera L. in the Arabidopsis $n d r 1$ mutant resulted in increased resistance of the transgenic plants to Botrytis cinerea by enhancing cell necrosis ${ }^{13}$. The expression of NHL1O in A. thaliana significantly increases after treatment with the cucumber mosaic virus $(\mathrm{CMV})$, suggesting that this gene may play a role in plant resistance ${ }^{14}$. In addition, the expression of AtNHL3 and AtNHL25 genes from A. thaliana is highly induced in response to pathogen infection, and AtNHL3 also responds rapidly to the derived signals of Pseudomonas syringae to generate a defense response ${ }^{15}$. Collectively, these findings strongly indicate that NHL proteins identified from different plant species are involved in triggering responses to a variety of biotic stresses and play an active role in inducing plant defense responses. However, the identification of $N H L$ genes from pepper $(C$. annuиm L.) and the functional characterization of the role of NHL genes in C. annuum disease resistance remain largely unknown. In this study, we aimed to identify the NHL genes in pepper in a genome-wide manner and understand the role of NHLs in pepper disease resistance, which will aid us in understanding the disease resistance of pepper.

\section{Results \\ Identification of CaNHL genes in C. annuum}

To identify the NHL genes in C. annuum, the sequence of the HIN1 gene from C. annuum was queried against the C. annuum genome via BLAST. The results of the BLAST search were then refined by removing the redundant sequences and through confirmation of the presence of the LEA-2 domain using SMART. Fifteen CaNHL genes were obtained, which were named CaNHL1-CaNHL15 based on the location within the reference genome (Table 1). To determine the chemical properties of these predicted CaNHL proteins, ProtParam tool (https://web.expasy.org/ protparam/) was used to calculate the molecular weight (MW), isoelectric point (pI), and chemical formula. Interestingly, we found that the MW of the vast majority of CaNHL protein ranges from 2000 to $3000 \mathrm{Da}$, with the exception of that of CaNHL12. The pI of all CaNHL proteins is between 9 and 10. In addition, the predicted three-dimensional structure of each protein is shown in Supporting Information 1.

\section{Phylogenetic analysis of NHL proteins within the Solanaceae}

To determine the evolutionary relationship among NHL proteins in Solanaceae species, we first retrieved the NHL protein sequences of Solanum lycopersicon (SINHL) and Nicotiana tabacum (NtNHL) from the Sol Genomics network (https://solgenomics.net/). An unrooted phylogenetic tree was subsequently constructed using the sequence alignment data of the 15 CaNHL proteins, 15 SINHL proteins, and 35 NtHIN1 proteins. As shown in Fig. 1, the 65 NHL proteins could be classified into five distinct groups. Group I includes CaNHL1, CaNHL2, CaNHL4, and CaNHL6; group II includes CaNHL3 and CaNHL7; group III includes CaNHL10, CaNHL11, and CaNHL12; group IV includes CaNHL8 and CaNHL15; and group V includes CaNHL5, CaNHL9, CaNHL13, and CaNHL14 (Fig. 1).

\section{Identification of regulatory elements and putative motifs in CaNHL proteins}

The promoter region of these CaNHL genes was predicted by TSSP (http://www.softberry.com/berry.phtml? topic $=$ tssp\&group $=$ programs\&subgroup $=$ promoter), and the regulatory elements in the promoter regions were predicted using information within the PlantCare database (http://bioinformatics.psb.ugent.be/webtools/plantcare/html/). The PlantCare-based analysis revealed that more than 26 regulatory elements are distributed within the promoters of the CaNHL genes. These elements include lightresponsive elements, defense response elements and 
Table 1 List of identified CaNHL genes in C. annuum.

\begin{tabular}{|c|c|c|c|c|c|c|c|}
\hline Name & Gene locus ID & Chr & Location & CDS (bp) & MW (Da) & pl & Formula \\
\hline CaNHL1 & Capana00g003650 & 1 & 63597266-63598081 & 816 & $29,899.83$ & 9.71 & $\mathrm{C}_{1342} \mathrm{H}_{2167} \mathrm{~N}_{363} \mathrm{O}_{385} \mathrm{~S}_{11}$ \\
\hline CaNHL2 & Capana02g000080 & 2 & 93168389-93169156 & 768 & $29,232.24$ & 9.98 & $\mathrm{C}_{1302} \mathrm{H}_{2114} \mathrm{~N}_{374} \mathrm{O}_{361} \mathrm{~S}_{14}$ \\
\hline CaNHL3 & Capana02g002134 & 2 & 150126019-150126702 & 684 & $25,936.06$ & 9.77 & $\mathrm{C}_{1169} \mathrm{H}_{1830} \mathrm{~N}_{328} \mathrm{O}_{319} \mathrm{~S}_{11}$ \\
\hline CaNHL4 & Capana02g002298 & 2 & $152229022-152229801$ & 780 & $29,408.50$ & 9.63 & $\mathrm{C}_{1320} \mathrm{H}_{2106} \mathrm{~N}_{366} \mathrm{O}_{360} \mathrm{~S}_{17}$ \\
\hline CaNHL5 & Capana03g001817 & 3 & $220067007-220067648$ & 642 & $23,985.79$ & 9.35 & $\mathrm{C}_{1104} \mathrm{H}_{1714} \mathrm{~N}_{278} \mathrm{O}_{307} \mathrm{~S}_{6}$ \\
\hline CaNHL6 & Capana03g001432 & 3 & $227321791-227322579$ & 789 & $29,461.79$ & 9.95 & $\mathrm{C}_{1325} \mathrm{H}_{2165} \mathrm{~N}_{367} \mathrm{O}_{363} \mathrm{~S}_{13}$ \\
\hline CaNHL7 & Capana04g002564 & 4 & 10211724-10212401 & 678 & $25,569.28$ & 9.97 & $\mathrm{C}_{1149} \mathrm{H}_{1788} \mathrm{~N}_{330} \mathrm{O}_{320} \mathrm{~S}_{7}$ \\
\hline CaNHL8 & Capana06g002149 & 6 & 158109052-158109642 & 591 & $22,370.18$ & 9.89 & $\mathrm{C}_{1016} \mathrm{H}_{1632} \mathrm{~N}_{276} \mathrm{O}_{279} \mathrm{~S}_{6}$ \\
\hline CaNHL9 & Capana06g000641 & 6 & $227434383-227435015$ & 633 & $23,626.44$ & 9.22 & $\mathrm{C}_{1084} \mathrm{H}_{1685} \mathrm{~N}_{283} \mathrm{O}_{295} \mathrm{~S}_{7}$ \\
\hline CaNHL10 & Capana10g002083 & 10 & $222022991-222023674$ & 684 & $26,003.05$ & 9.09 & $\mathrm{C}_{1167} \mathrm{H}_{1819} \mathrm{~N}_{317} \mathrm{O}_{329} \mathrm{~S}_{14}$ \\
\hline CaNHL11 & Capana10g002082 & 10 & $222083709-222084392$ & 684 & $25,999.04$ & 9.13 & $\mathrm{C}_{1169} \mathrm{H}_{1823} \mathrm{~N}_{317} \mathrm{O}_{329} \mathrm{~S}_{13}$ \\
\hline CaNHL12 & Capana10g002081 & 10 & $222108128-222108451$ & 324 & $12,528.35$ & 9.27 & $\mathrm{C}_{556} \mathrm{H}_{878} \mathrm{~N}_{158} \mathrm{O}_{162} \mathrm{~S}_{5}$ \\
\hline CaNHL13 & Capana10g002080 & 10 & $222113163-222113798$ & 636 & $23,953.74$ & 9.67 & $\mathrm{C}_{1095} \mathrm{H}_{1702} \mathrm{~N}_{298} \mathrm{O}_{295} \mathrm{~S}_{6}$ \\
\hline CaNHL14 & Capana11g000361 & 11 & $248346066-248346710$ & 645 & $23,881.81$ & 9.06 & $\mathrm{C}_{1098} \mathrm{H}_{1726} \mathrm{~N}_{284} \mathrm{O}_{301} \mathrm{~S}_{5}$ \\
\hline CaNHL15 & Capana11g000183 & 11 & 254198867-254199487 & 621 & $23,494.29$ & 9.90 & $\mathrm{C}_{1072} \mathrm{H}_{1677} \mathrm{~N}_{297} \mathrm{O}_{286} \mathrm{~S}_{6}$ \\
\hline
\end{tabular}

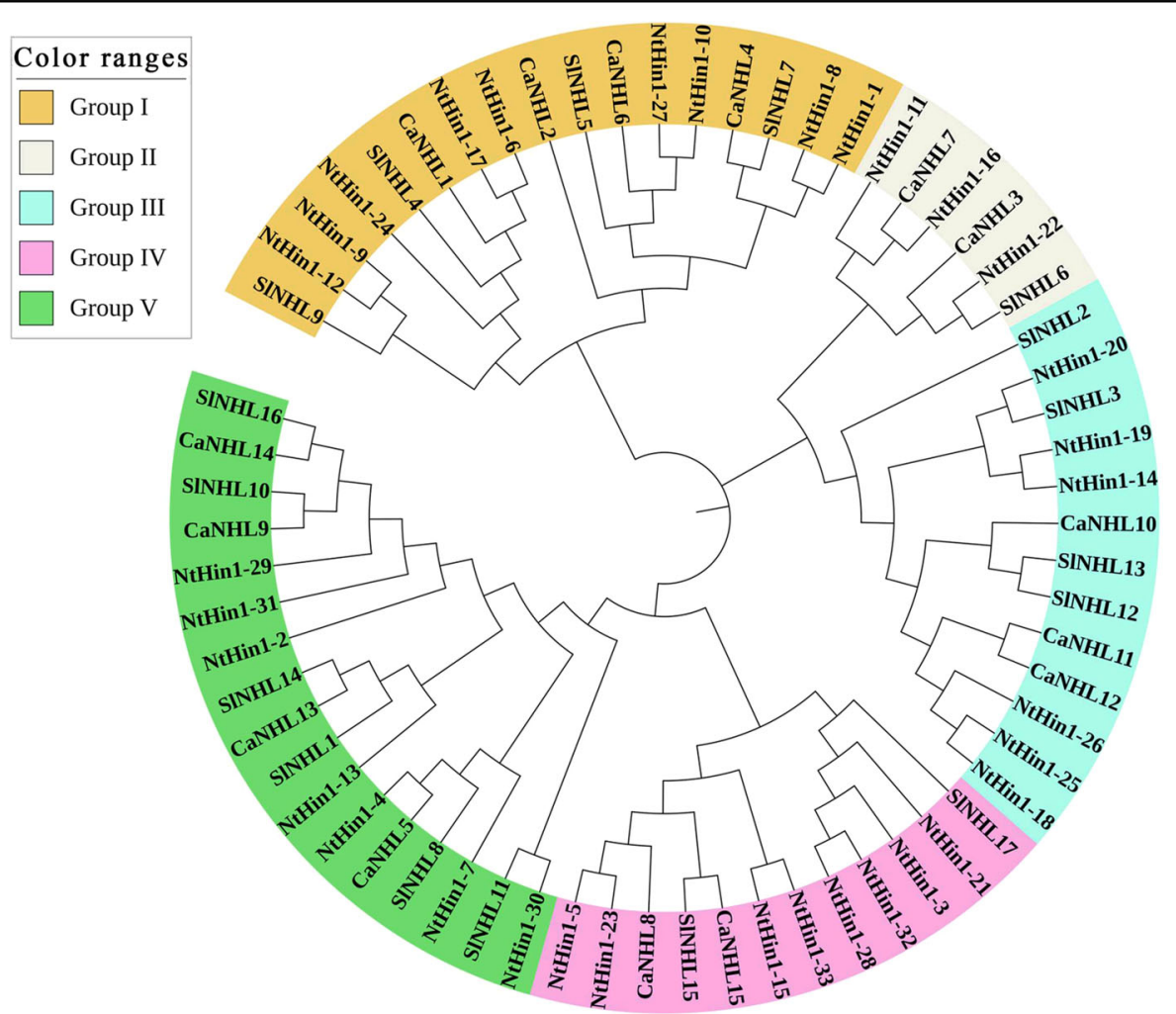

Fig. 1 Unrooted phylogenetic tree of NHL protein family from C. annuum, S. lycopersicon, and N. tabacum MEGA 7.0.26 was used to construct thephylogenetic tree based on the NHL protein sequences, and the five distinct subgroups of NHL proteins are shown. iTOL was used to annotate and review the phylogenic tree. 
hormone induction elements, such as those for abscisic acid (ABA), auxin (IAA), gibberellin (GA), and SA (Fig. 2a). In addition, we found that the promoters of CaNHL2, CaNHL4, CaNHL7, CaNHL8, CaNHL9, and CaNHL10 contain many methyl jasmonate (MeJA)-responsive elements, suggesting that these genes might be involved in the JA-signaling pathway. Thus, these observations strongly suggest that the role of NHL proteins in pepper is likely associated with disease resistance. MEME motif analysis revealed that motifs 2 and 3 are widely distributed in all CaNHL proteins (Fig. 2b). Interestingly, we found that CaNHL members within the same phylogenetic group share a similar motif composition. For example, motif 1 , motif 2, motif 3, and motif 4 are present within CaNHL8 and CaNHL15, which are located on the same branch (Fig. 2b). The similar motif arrangements among the CaNHL proteins indicate that the protein architecture is conserved within specific subfamilies. However, the functions of most of these conserved motifs remain to be elucidated. Overall, our results suggest that CaNHL proteins in the same phylogenic group share conserved motifs and similar protein domain compositions, suggesting that they have similar functions in plant development and disease resistance.

Genome-wide transcriptomic analysis of $\mathrm{CaNHL}$ genes and quantification of CaNHL genes under exogenous MeSA and MeJA treatments

To determine the global expression profiles of all CaNHL genes in different plant tissues and in response to various stresses, we analyzed the published RNA-seq data available in the Pepper Informatics Hub (http:// pepperhub.hzau.edu.cn/) database and generated a heat map of all the CaNHL genes (Fig. 3). Figure 3a shows that CaNHL10, CaNHL11, and CaNHL13 displayed constitutive expression in all examined plant tissues, including the leaves, stems, roots, flowers, petals, stamens, and fruits. However, some CaNHL genes exhibited tissuespecific expression patterns, and some did not show any expression. CaNHL9 is expressed specifically in the flowers, and the expression of CaNHL3, CaNHL6, CaNHL7, and CaNHL8 was not detected in any tested plant tissues. When pepper plants were challenged with different abiotic stresses, such as cold and heat, and

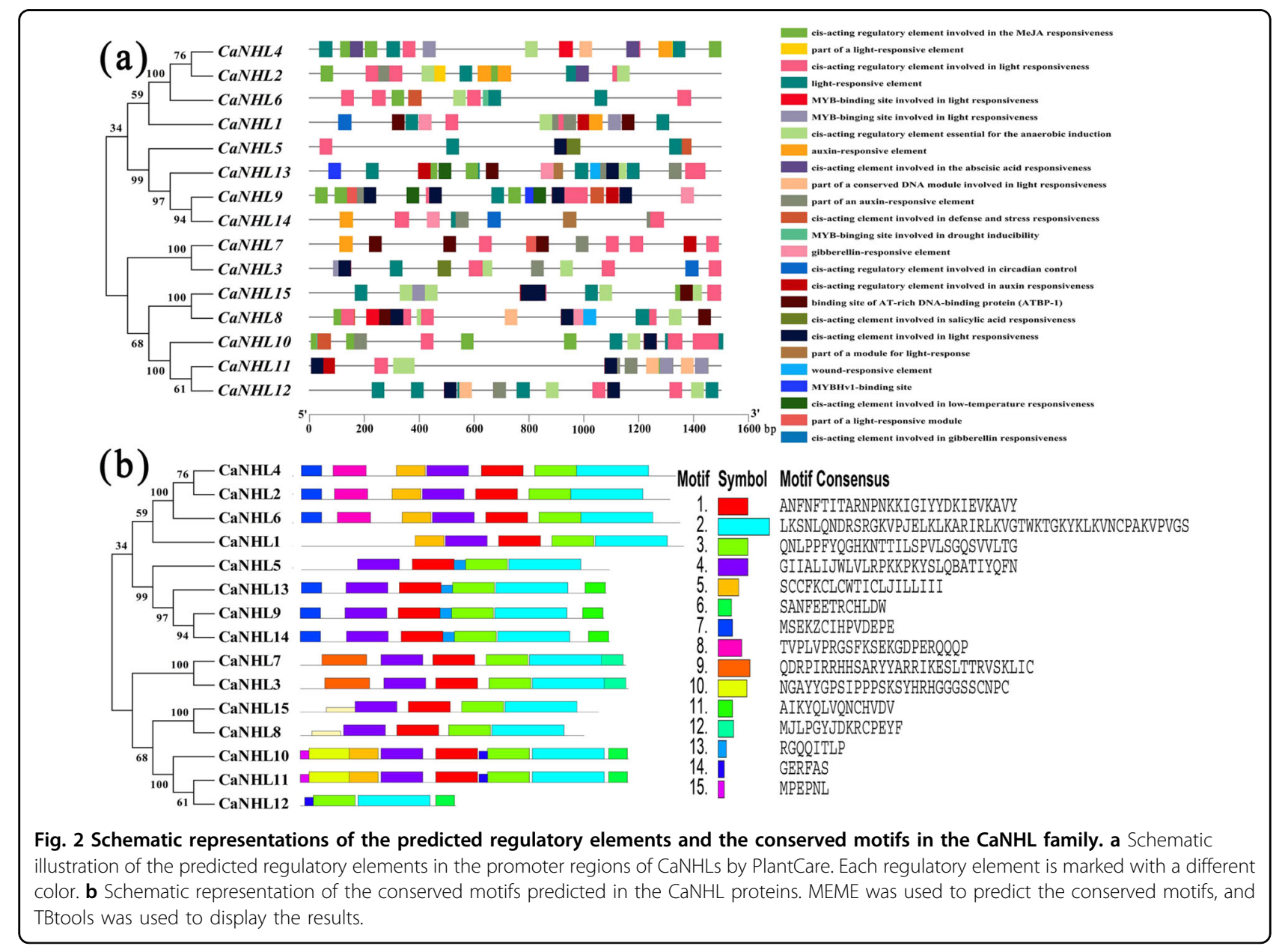


(a)

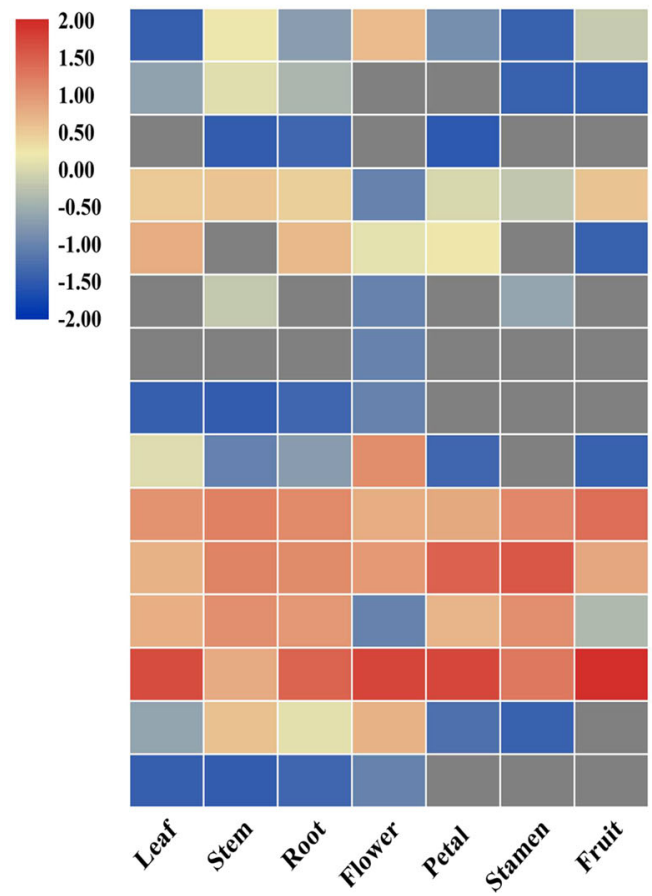

(b)

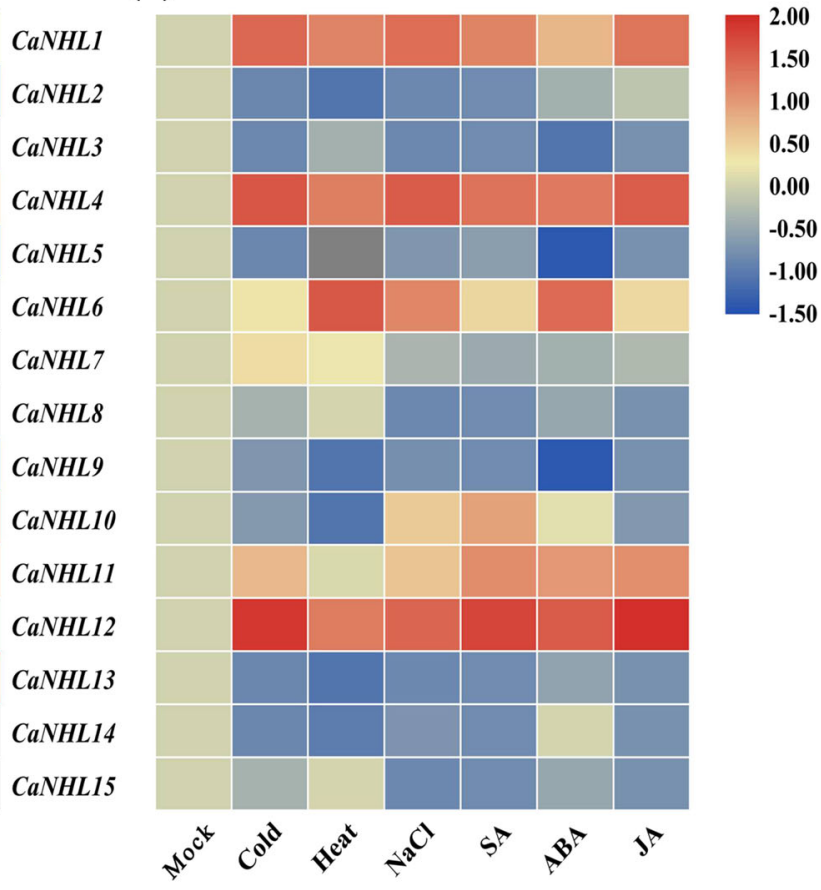

Fig. 3 Heat map showing differential expression of CaNHL genes based on an RNA-seq transcriptomic analysis. a Expression of CaNHL genes in different pepper tissues. The columns represent the leaves, stems, roots, flowers, petals, stamens, and fruits, from left to right. $\mathbf{b}$ Expression profiles of $\mathrm{CaNHL}$ genes in response to various stresses. From left to right, the columns indicate the mock; abiotic stresses such as cold, heat, and $\mathrm{NaCl}$; and hormone treatments such as SA, ABA, and JA.

subjected to $\mathrm{NaCl}, \mathrm{SA}, \mathrm{ABA}$, and JA treatments, differential expression of $\mathrm{CaNHL}$ genes was observed (Fig. 3b). CaNHL1, CaNHL4, and CaNHL12 were highly upregulated in response to all the stresses, and CaNHL6 was specifically and highly induced in the plants subjected to heat and $A B A$ treatments, which suggests that some of the $\mathrm{CaNHL}$ genes are involved in abiotic stress responses.

To validate the results obtained from the transcriptomic data, qPCR analysis was employed to quantify the differentially expressed $\mathrm{CaNHL}$ genes at $0,3,6$, and $12 \mathrm{~h}$ postexogenous MeJA and methyl salicylate (MeSA) treatment. MeSA is a derivative of SA, and the accumulation of MeJA and MeSA coincides with the accumulation of endogenous $\mathrm{JA}$ and $\mathrm{SA}^{16-18}$. Figure 4 shows that the expression of CaNHL1, CaNHL4, CaNHL6, CaNHL10, CaNHL11, and CaNHL12 is highly induced after MeJA or MeSA treatment, which is consistent with the RNA-seq data. However, the expression pattern of individual $\mathrm{CaNHL}$ genes at specific time points after treatment is different. After MeJA treatment, the expression of CaNHL1 increased and peaked at the $3 \mathrm{~h}$ time point, decreased at $6 \mathrm{~h}$, and then increased again at the $12 \mathrm{~h}$ time point. The expression of CaNHL4, CaNHL10, and CaNHL11 increased but then decreased, while the expression of CaNHL6 and CaNHL12 continued to increase and peaked at the $12 \mathrm{~h}$ time point after treatment (Fig. 4). After the application of MeSA, the expression of CaNHL4 increased at the $3 \mathrm{~h}$ time point, after which it decreased but then peaked at the $12 \mathrm{~h}$ time point. The expression of the remaining five genes increased first, peaked at the 3 or $6 \mathrm{~h}$ time point and then decreased at the $12 \mathrm{~h}$ time point. Taken together, our findings demonstrate that six $\mathrm{CaNHL}$ genes are highly activated by MeSA and MeJA treatments.

\section{Expression of CaNHL genes in response to $P$. capsici, $P$. syringae, and TMV infection}

To further assess the expression profiles of $\mathrm{CaNHL}$ genes in response to biotic stresses, we inoculated pepper plants with $P$. capsici, $P$. syringae, and TMV. Total RNA was extracted from samples collected at 0,24 , and $48 \mathrm{~h}$ post- $P$. capsici inoculation and at 2,4 , and $6 \mathrm{~d}$ after $P$. syringae and TMV inoculation. As shown in Fig. 5a, the CaNHL4, CaNHL6, and CaNHL12 genes were significantly induced at 24 hpi with $P$. capsici, while the expression patterns of CaNHL1, CaNHL10, and CaNHL11 were similar to those of the mock plants. Interestingly, at $48 \mathrm{hpi}$, the expression level of all six genes was significantly higher than that in the mock plants (Fig. 5a). After P. syringae inoculation, the CaNHL1, CaNHL4, 


\section{MeJA Treatment}

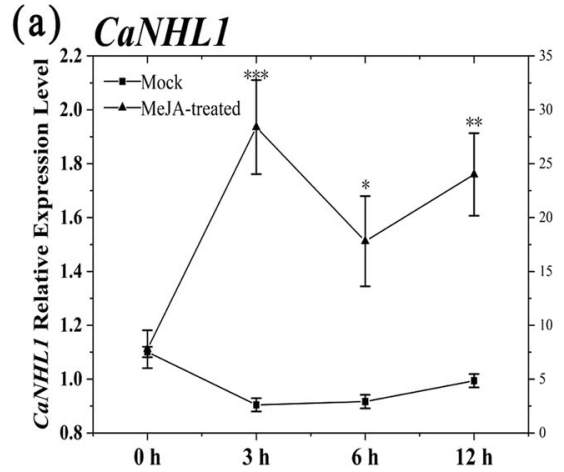

(d) CaNHL10

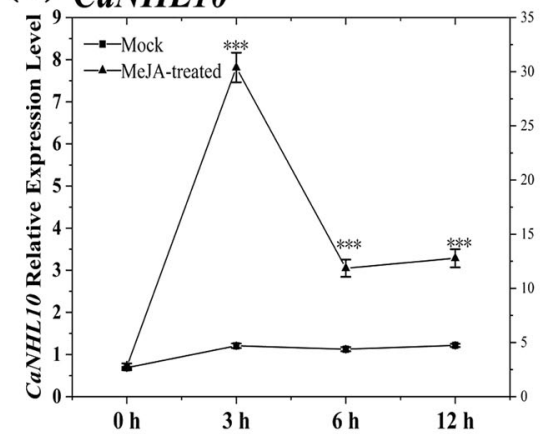

(g) CaNHL1

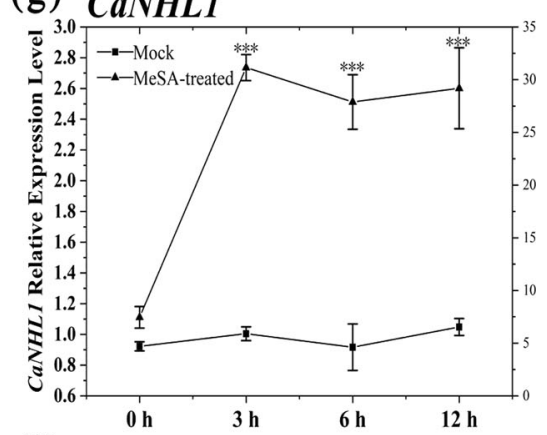

(j) CaNHL10

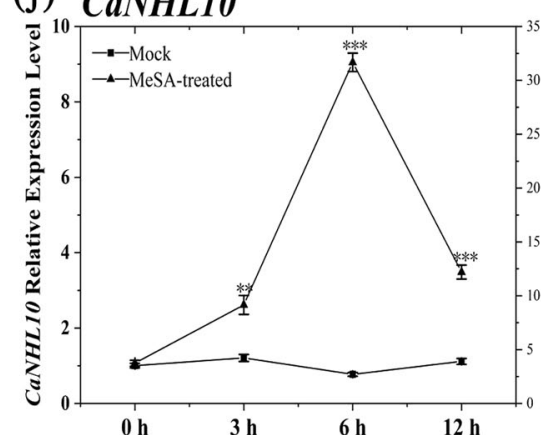

(b) $\mathrm{CaNHL} 4$

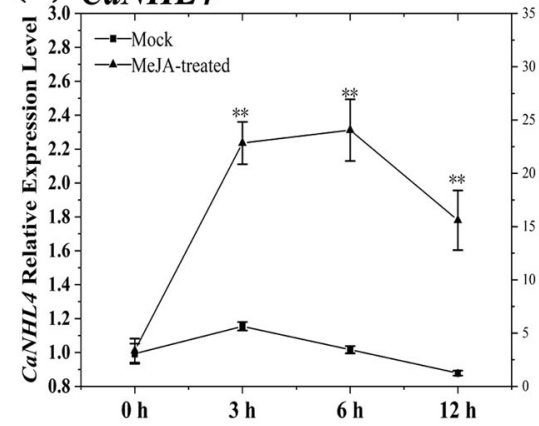

(e) CaNHL11

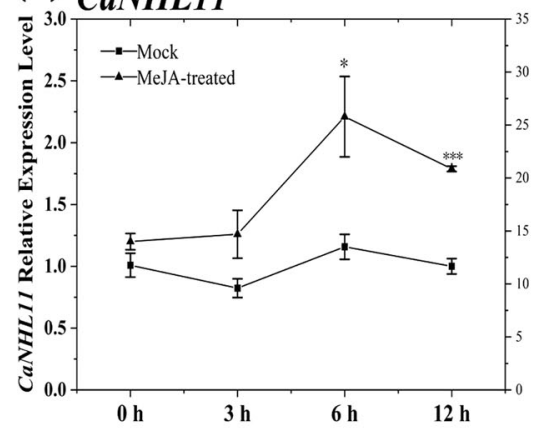

MeSA Treatment

(h) CaNHL4

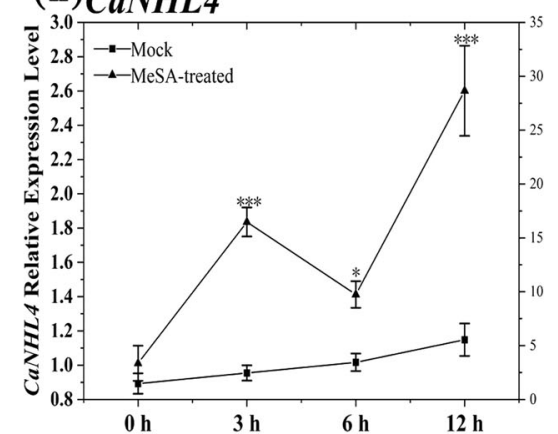

(k) CaNHL11

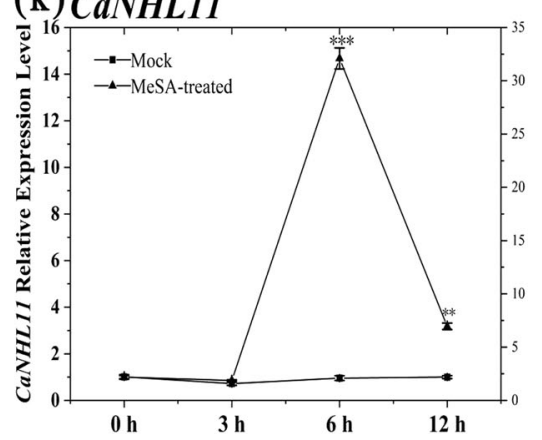

(c) CaNHL6

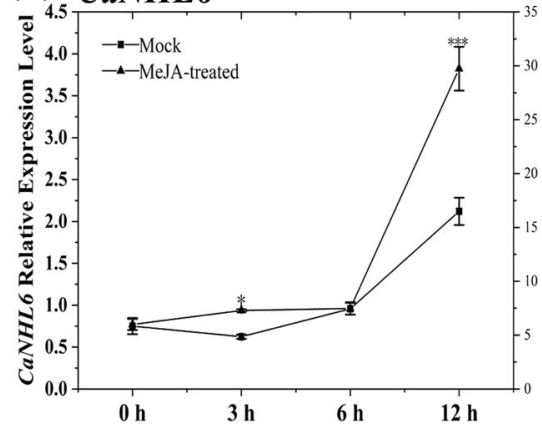

(f) CaNHL12

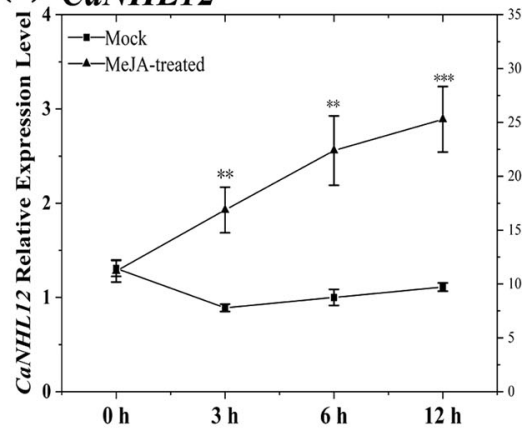

(i) CaNHL6

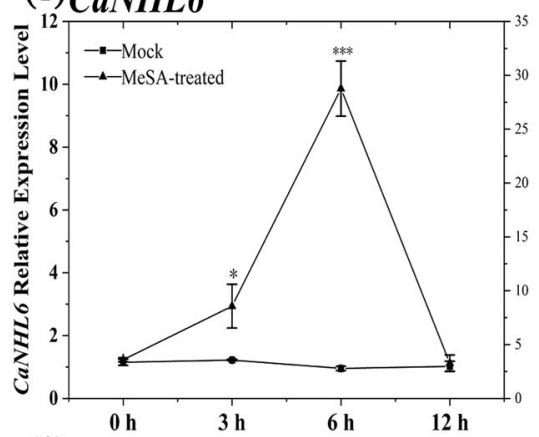

(1) CaNHL12

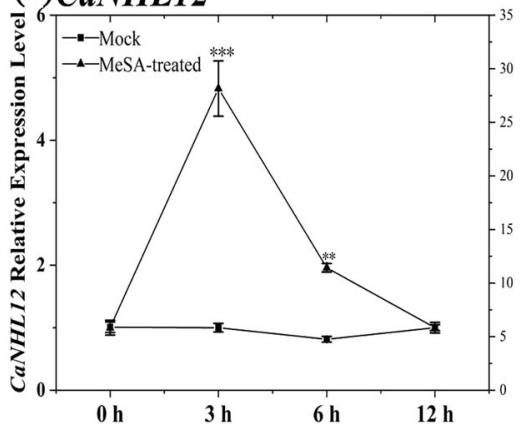

Fig. 4 Relative expression of the CaNHL1, CaNHL4, CaNHL6, CaNHL10, CaNHL11, and CaNHL12 genes in pepper leaves after MeJA and MeSA treatments. a-f Expression level of CaNHL1, CaNHL4, CaNHL6, CaNHL10, CaNHL11, and CaNHL12 at 3, 6, 12 h after MeJA treatment. g-I Expression level of the above-mentioned CaNHL genes at the 3,6,12 h time points after MeSA treatment. The values represent the means \pm standard errors (SEs) of three biological replications. The statistical analyses were performed using Student's $t$-test $(* 0.01<P<0.05 ; * * 0.001<P<0.01 ; * * * P<$ 0.001). "Mock" indicates plants without MeJA or MeSA treatment. 


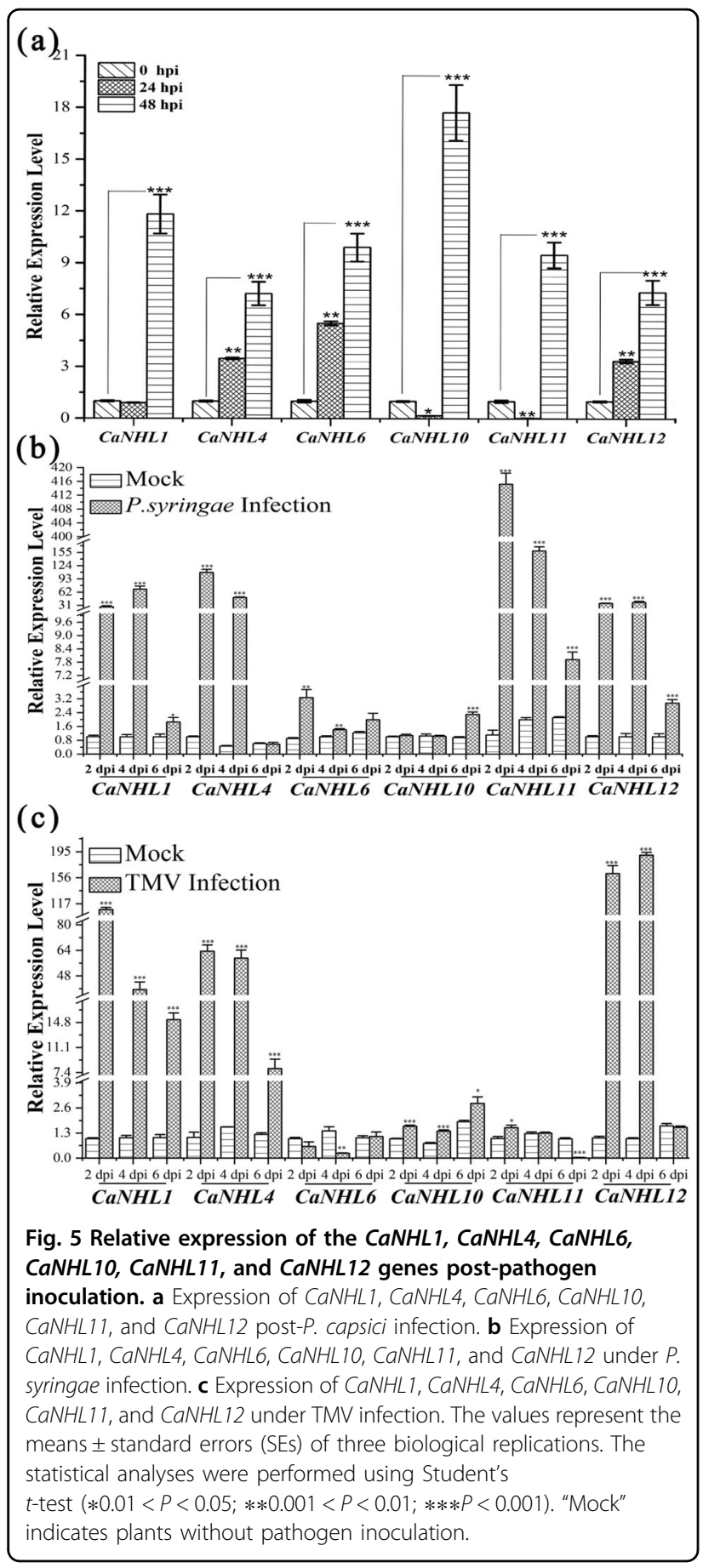

CaNHL11, and CaNHL12 genes were significantly induced at 2, 4, and $6 \mathrm{dpi}$ in comparison to those in the mock plants, while the expression of CaNHL1O was similar to that in the mock plants at 2 and 4 dpi (Fig. 5b). Figure 5c shows that after TMV inoculation, the expression of the CaNHL genes, especially CaNHL1, CaNHL4, CaNHL10, and CaNHL12, was significantly induced at 2 $\mathrm{dpi}$; however, the expression of CaNHL6 was not induced.
At 4 and $6 \mathrm{dpi}$, the expression level of most genes tended to decrease, among which CaNHL6 and CaNHL11 showed lower expression than did those in the mock plants. Overall, these findings strongly indicated that the CaNHL1, CaNHL4, and CaNHL12 genes are highly induced in response to infection by these three pathogens, suggesting that they might play a significant role in the defense response against them.

\section{Silencing CaNHL4 in pepper increases susceptibility to different pathogens}

As CaNHL4 is highly induced in pepper plants subjected to MeSA and MeJA treatments and in response to pathogen infection, we decided to silence CaNHL4 to examine the effects of CaNHL4 silencing in pepper upon pathogen infection. We utilized the virus-induced gene silencing (VIGS) approach to silence CaNHL4 (TRV: CaNHL4), and TRV:00 served as a mock. To determine the silencing efficacy of our designed constructs, the number of CaNHL4 and TRV-CP transcripts was quantified by qPCR at $14 \mathrm{~d}$ after TRV inoculation. Figure $6 \mathrm{a}$ shows that the expression level of CaNHL4 was significantly decreased relative to that of the mock plant, and no significant difference was observed in the accumulation of TRV-CP transcripts, indicating that the silencing efficacy of CaNHL4 was sufficient for our subsequent experiments. We then simultaneously inoculated the silenced plants and mock plants with $P$. capsici, and disease development was examined at $2 \mathrm{dpi}$. As expected, larger disease lesions were observed on the silenced plants than on the mock plants, indicating that silencing CaNHL4 enhances P. capsici infection (Fig. 6b, c). In addition, we inoculated the silenced plants and mock plants with TMV:GFP. Figure $6 \mathrm{~d}$ shows that pronounced GFP signals were observed on the leaves of the silenced plants compared to the leaves of the mock plants at 2 and $4 \mathrm{dpi}$ after TMV inoculation. At 7 and $9 \mathrm{dpi}$, the mosaic disease symptoms observed on the young leaves of the silenced plants developed rapidly compared to those on the mock plants. Furthermore, the number of TMV:GFP transcripts and accumulation of TRV-GFP proteins in inoculated and young leaves of the silenced plants and mock plants were quantified by qPCR and western blots, respectively. Figure $6 \mathrm{e}, \mathrm{f}$ show that the expression level of GFP and the accumulation of TRV-GFP proteins in the silenced plants were significantly higher than those in the mock plants. These findings suggested that silencing CaNHL4 enhances the infection and movement of TMV: GFP. Similarly, we inoculated the silenced plants and mock plants with $P$. syringae; the disease development was monitored, and lesion size was measured at 2, 4, and 6 dpi. Figure $6 \mathrm{~g}$ shows that the development of the disease symptoms on the leaves of the silenced plants was significantly faster than that of the mock plants, and the 


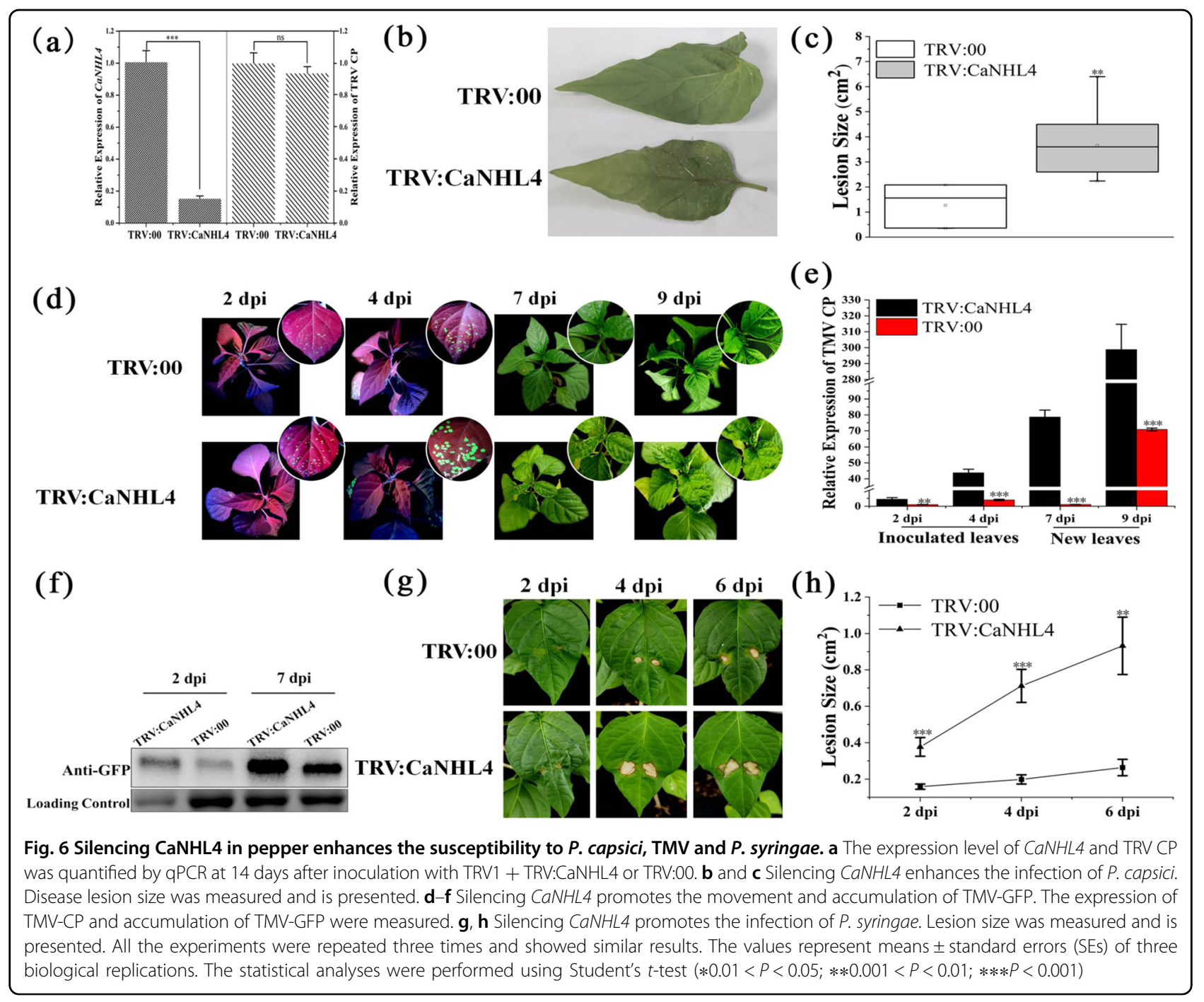

lesion size on the silenced plants was significantly larger than that of the mock plants (Fig. 6h), suggesting that silencing CaNHL4 increases the infection of P. syringae. Taken together, our findings reveal that silencing CaNHL4 significantly enhances the susceptibility of pepper to infection by the three pathogens, suggesting that CaNHL4 participates in the defense response against pathogens.

\section{Overexpression of CaNHL4 in pepper enhances resistance to different pathogens}

To further understand the function of CaNHL4 in defense against different pathogens, we generated a pART27-N-7::Myc-CaNHL4 expression vector and transiently overexpressed it in the leaves of pepper $(C$. annuum) by agroinfiltration, with a Myc:00 empty vector serving as the mock. At $2 \mathrm{dpi}$, western blotting was used to detect the accumulation of Myc-CaNHL4 protein. As shown in Fig. 7a, Myc-CaNHL4 proteins were detected in the infiltrated leaves. We inoculated P. capsici, P. syringae, and TMV on the infiltrated leaves at $2 \mathrm{~d}$ after infiltration. The disease development on the inoculated leaves was monitored and visualized via trypan blue staining. Figure $7 \mathrm{~b}, \mathrm{c}$ shows that overexpression of Myc:CaNHL4 attenuated the disease development of $P$. capsici, as evidenced by reduced lesion size after trypan blue staining. Similarly, limited GFP signals were detected in the leaves overexpressing CaNHL4 compared to mock leaves overexpressing the Myc:00 empty vector at 4, 5, and 6 dpi after TMV:GFP inoculation (Fig. 7d). The number of TMV CP transcripts in the leaves overexpressing CaNHL4 was significantly lower than that in the mock plants at 4 and $6 \mathrm{dpi}$ (Fig. 7e). Western blot analysis showed that the accumulation of TMV:GFP at 4 dpi was lower than that of the mock plants (Fig. 7f), indicating that overexpression of 
(a)

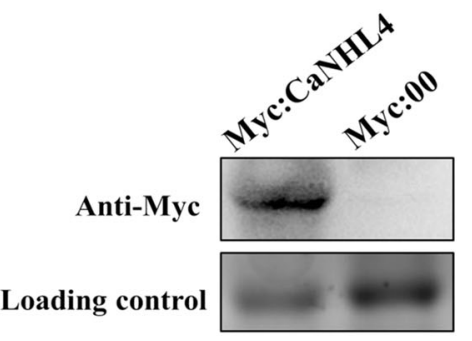

(d)

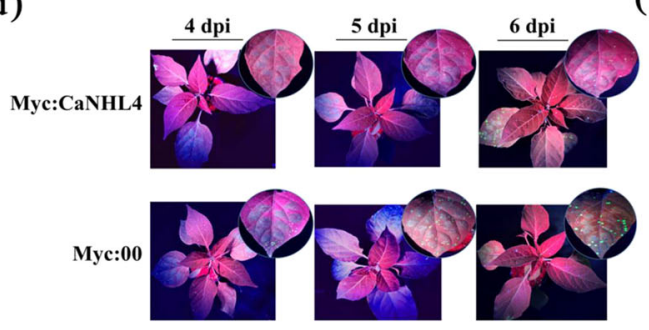

$(\mathrm{g})$

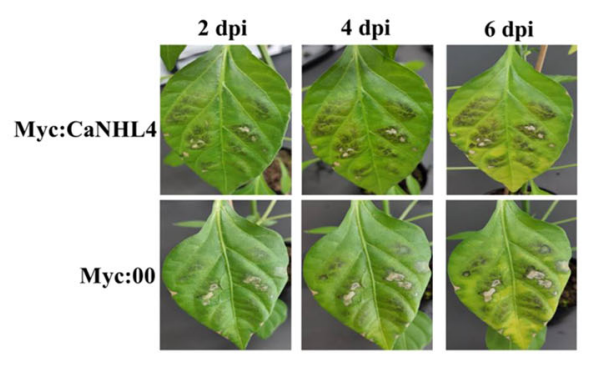

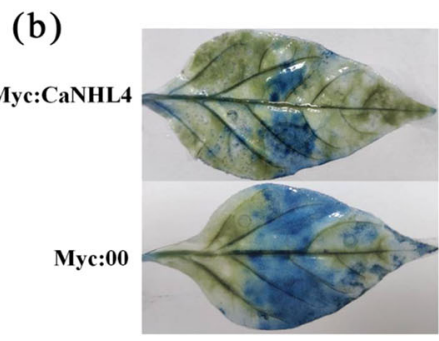

(e)

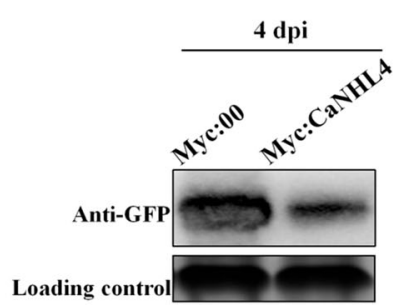

(h)

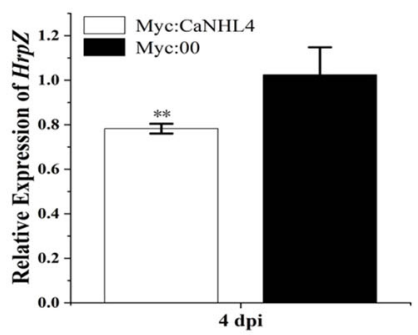

(c)

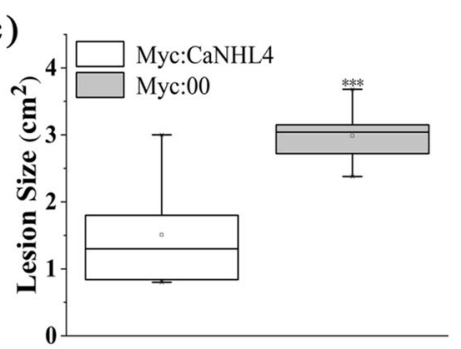

(f)

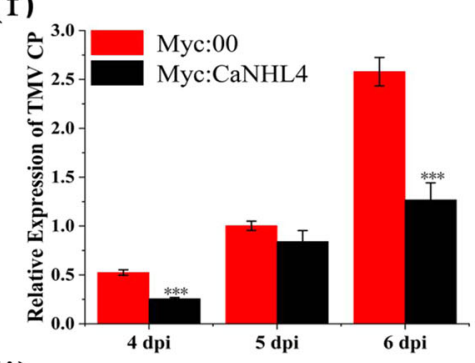

(i)

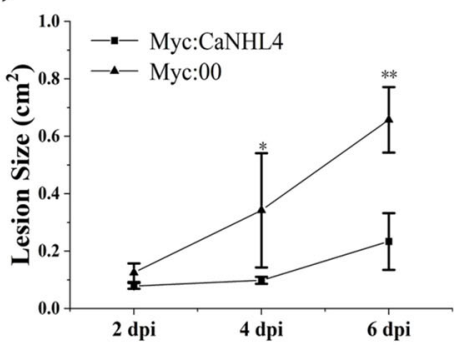

Fig. 7 Transient overexpression CaNHL4 in pepper enhances resistance to $P$. capsici, TMV, and $P$. syringae. a The accumulation of MycCaNHL4 was quantified by western blotting at $2 \mathrm{~d}$ after agroinfiltration. A Myc:00 empty vector was used as a mock. $\mathbf{b}$ and $\mathbf{c}$ Transient overexpression of CaNHL4 decreases the infection of P. capsici. Disease lesions were stained with trypan blue and measured. $\mathbf{d}-\mathbf{f}$ Transient overexpression of CaNHL4 inhibits the infection of TMV-GFP. The accumulation of TMV-GFP in CaNHL4 overexpressing leaves was reduced. The number of TMV CP transcripts significantly decreased, and the accumulation of My-CaNHL4 protein also decreased. $\mathbf{g}$-i Transient expression of CaNHL4 decreases the infection of $P$. syringae. The disease development of $P$. syringae is presented, and the lesion size was measured at 2, 4, and 6 dpi. The expression of $H r p Z$ gene was quantified by qPCR, revealing bacterial mass in the infected leaves at $4 \mathrm{dpi}$. The HrpZ gene expression levels were normalized to the expression of pepper actin. All experiments were repeated three times and showed similar results. The values represent the means \pm standard errors (SEs) of three biological replications. The statistical analyses were performed using Student's $t$-test $(* 0.01<P<0.05 ; * * 0.001<P$

$<0.01 ; * * * P<0.001)$.

CaNHL4 compromises the replication of TMV:GFP. After $P$. syrinage inoculation, the disease lesions were observed at 2, 4, and 6 dpi. Figure $7 \mathrm{~g}$, h show that the disease development of P. syringae on CaNHL4-overexpressing leaves was significantly slower than that on the mock leaves. Furthermore, we performed qPCR to quantify the expression of $H r p Z$, a pathogenic gene from P. syringae, to confirm the growth of $P$. syringae. As shown in Fig. 7i, at $4 \mathrm{dpi}$, the expression level of $\mathrm{HrpZ}$ in the CaNHL4overexpressing leaves was significantly lower than that in the mock leaves, indicating that transient overexpression of CaNHL4 reduces the infection of $P$. syringae. Taken together, the results show that silencing CaNHL4 increases the susceptibility of pepper to these three pathogens but that transient overexpression of CaNHL4 improves the resistance of pepper to these three pathogens, suggesting that CaNHL4 contributes to the resistance of pepper against different pathogens.

\section{CaNHL4 localizes to the plasma membrane}

To explore the subcellular localization of CaNHL4, we generated a CaNHL4:mGFP construct encoding a CaNHL4 protein fused to mGFP. The plasma membrane marker dsRFP was used as a positive control. Agrobacterium tumefaciens carrying either CaNHL4:mGFP or $P M$-Marker-dsRFP was coinfiltrated into the leaves of Nicotiana benthamiana plants. Two days after infiltration, green fluorescent signals were visualized using confocal microscopy, and CaNHL4-mGFP was found to be localized in the plasma membrane as it colocalized with the red fluorescent signals of the plasma membrane marker PM-Marker-dsRFP (Fig. 8). 


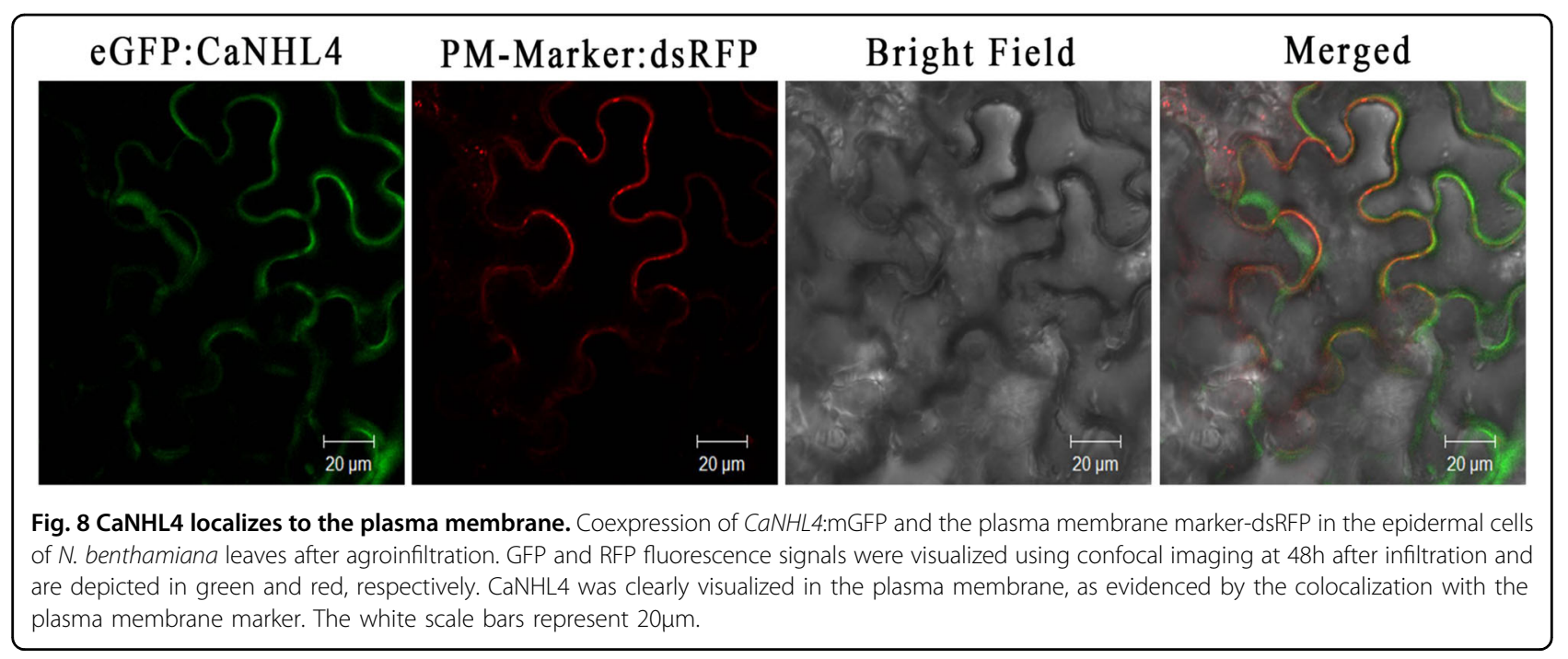

CaNHL4 participates in the antistress defense of pepper by mediating the SA and JA signaling pathways and ROS production

To further determine the effects of CaNHL4 on the SA and JA signaling pathways, we examined the expression of SAresponsive genes, including non-expresser of pathogenesisrelated gene 1 (NPR1), pathogenesis-related gene 1 (PR1), pathogenesis-related gene $2(P R 2)^{19}$, JA-responsive gene $M Y C 1 \alpha$, JA receptor coronatine insensitive 1 (COI1), and ethylene response factor $1(E R F 1)^{20,21}$, in CaNHL4-silenced leaves and CaNHL4-overexpressing leaves. Surprisingly, we found that the expression of these genes in the silenced plants decreased significantly (Fig. 9a), suggesting that silencing CaNHL4 compromises the expression of these genes, resulting in the deactivation of the SA and JA signaling pathways. Similarly, we found that the expression level of the ROS-induced gene glutathione S-transferase 6 (GST6) ${ }^{22}$ was significantly decreased and that ROS production was significantly reduced in the silenced plants compared to that in the mock plants, as revealed by DAB staining, suggesting that CaNHL4 is also involved in ROS production (Fig. 9a, b). However, overexpressing CaNHL4 significantly increased the expression of SA-responsive and JA-responsive genes and the ROS-induced gene GST6 (Fig. 9a), and increased ROS production was observed (Fig. 9c). In conclusion, our results indicated that CaNHL4 affects the expression of SAresponsive and JA-responsive genes and ROS production, suggesting that CaNHL4 might be involved in the antistress defense response of pepper by activating the SA and JA signaling pathways and ROS production.

\section{Discussion}

It has been documented that $N H L$ genes are involved in the process of plant disease resistance and stress tolerance in Arabidopsis, tobacco, soybean, grape, tomato, and other plant species $^{23}$. In our study, we performed genome-wide identification of NHL genes in pepper. Functional analysis of the CaNHL genes revealed that CaNHL4 plays an important role in the disease resistance of pepper.

On the basis of bioinformatic analysis, our results showed that members of CaNHL family contain the conserved LEA-2 domain. Previous studies have shown that members of the LEA protein family are involved in antistress responses ${ }^{24-26}$. For example, the gene encoding LEA-containing proteins is highly expressed in the late embryonic development of seeds and is highly expressed under environmental stresses, such as drought and low temperature $^{24}$. Zegzouti et al. reported that the LEA-like protein ER5 is expressed in response to drought, ABA, and injury ${ }^{25}$. Singh et al. found that the expression of LEA14 in A. thaliana is induced under stress conditions, such as drought, coldness, osmotic stress, and high temperature $^{26}$. Our findings revealed that some CaNHL genes encoding LEA-containing proteins are induced under stress conditions, such as cold, high temperature, ABA, JA, and SA treatments (Fig. 3), which is consistent with the results of previous studies. These findings suggest that members of the CaNHL protein family may be involved in the disease resistance of pepper.

Regulatory element analysis showed that the promoter regions of $\mathrm{CaNHL}$ genes contains many stress-responsive and defense-responsive-related elements, such as those involving MeJA, SA, and the drought response (Fig. 2a), suggesting that CaNHLs might participate in the pepper defense response. Many studies have shown that NHLs participate in the JA-mediated and ET-mediated defense response pathways ${ }^{27}$. In pepper, CaNHL9 and CaNHL10 contain 10 MeJA-responsive elements, CaNHL4 has six MeJA-responsive elements, and the remaining genes, including CaNHL11 and CaNHL15, also have MeJAresponsive elements (Fig. 2a). These findings suggest that 

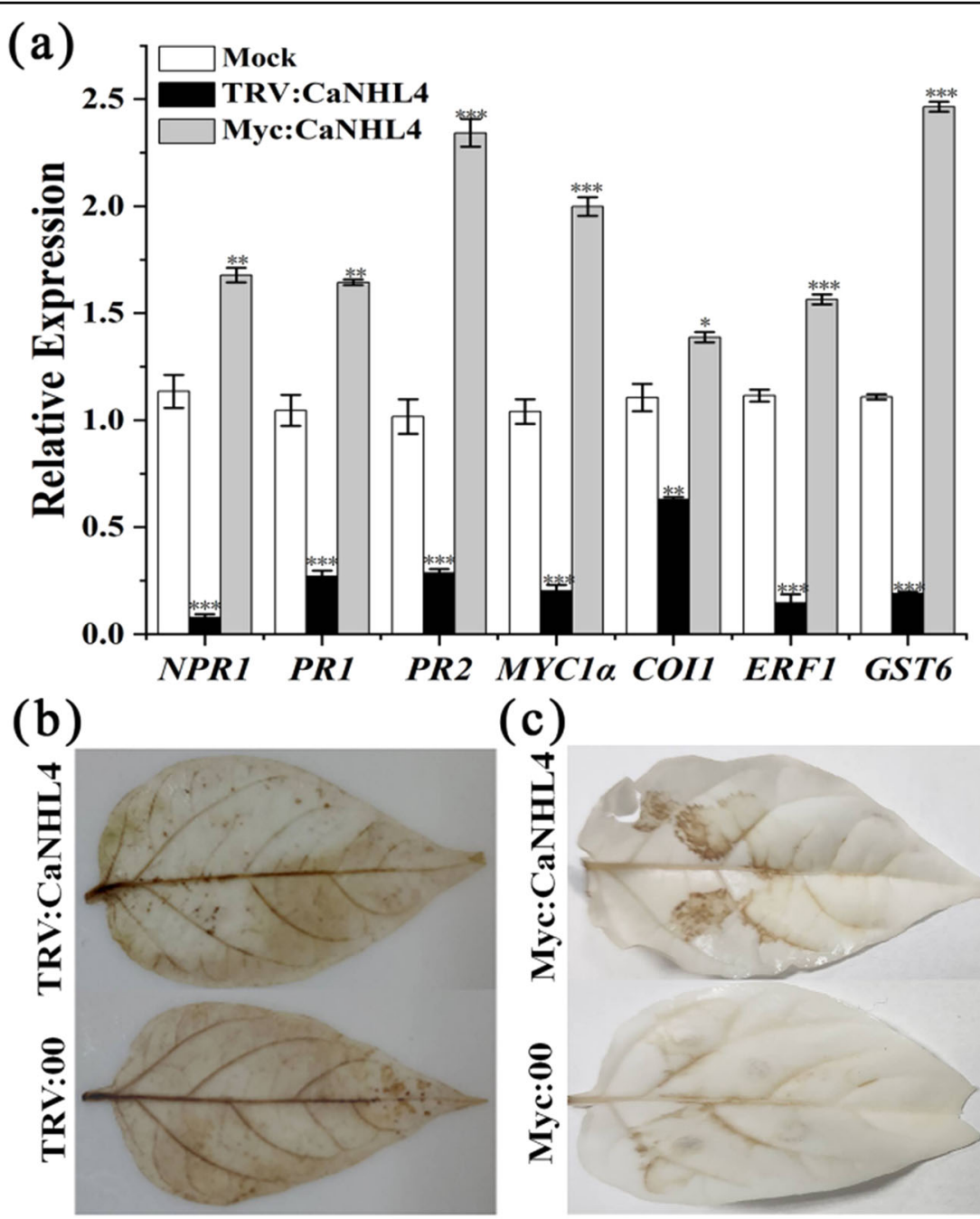

(c)

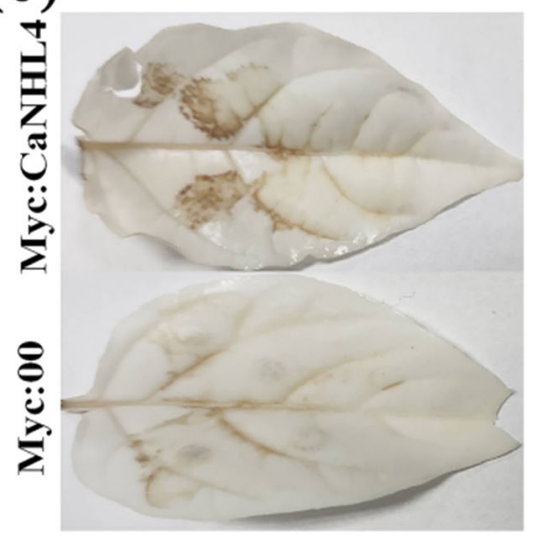

Fig. 9 Silencing CaNHL4 reduces the expression of SA-responsive, JA/ET-responsive and ROS-responsive genes, and ROS production, whereas transient overexpression of CaNHL4 yields the opposite effects. a Effects of silencing and transient overexpression of CaNHL4 on the expression of the SA-responsive genes NPR1, PRI, and PR2; the JA/ET-related genes MYC1a, COI1, and ERF1; and the ROS-induced gene GST6. b Effects of silencing CaNHL4 on ROS production. c Effects of transiently overexpressing CaNHL4 on ROS production. DAB staining was used to detect the ROS production. All the experiments were repeated three times and showed similar results. The values represent the means \pm standard errors (SEs) of three biological replications. The statistical analyses were performed using Student's $t$-test $(* 0.01<P<0.05, * * 0.001<P<0.01, * * * P<0.001)$.

some CaNHL genes might play a role in disease resistance by mediating the JA-signaling pathway. However, cisacting elements of SA also are present within the promoter of CaNHL4, indicating that this gene might also participate in the SA-responsive signaling pathway. RNASeq and qPCR analysis showed that $C a N H L$ genes are highly induced after exogenous MeJA and MeSA treatments (Figs. 3 and 4). In addition, we found that the expression of the JA/ET-responsive genes $M Y C 1 \alpha, C O I 1$, and ERF1 and the SA-responsive genes NPR1, PR1, and $P R 2$ was also inhibited in CaNHL4-silenced plants (Fig. 9), providing strong evidence that CaNHL4 is involved in stress resistance by mediating the JA and SA signaling pathways. Interestingly, we also found that many ABA- responsive elements are present in the promoter regions of CaNHL genes (Fig. 2a). Previous studies have shown that the gene encoding LEA-containing proteins is also induced in response to $\mathrm{ABA}^{25}$. In addition, $\mathrm{ABA}$ treatment of $A$. thaliana can upregulate SA-induced sesquiterpene synthesis, which indirectly indicates that $\mathrm{ABA}$ may participate in SA-mediated defense ${ }^{28}$. $\mathrm{ABA}$ is able to induce the expression of defense-related genes ${ }^{29}$, especially those that enhance plant resistance to viruses ${ }^{30,31}$. However, the role of ABA-responsive elements present in the promoter regions of CaNHLs remains unclear. We also found that the promoter region of each CaNHL gene contains light-responsive elements (Fig. 2), so we speculated that $C a N H L$ genes might be induced by light. Our 
findings suggested that the activation of some CaNHL genes is not strongly correlated with the predicted ciselements in the promoter region (Supporting Information 2). For example, we found that the promoters of both CaNHL4 and CaNHL6 contain SA-responsive elements. Thus, we hypothesized that CaNHL4 and CaNHL6 could be induced by SA treatment, which is in agreement with our findings that both CaNHL4 and CaNHL6 are induced by SA treatment (Fig. 4). Conversely, the promoter regions of CaNHL2, CaNHL4, CaNHL6, CaNHL8, CaNHL10, CaNHL11, and CaNHL15 contain MeJAresponsive elements (Fig. 2a), indicating that these genes might be induced in response to MeJA. However, different expression patterns of these genes were observed on the basis of the RNA-seq data and qPCR analysis (Figs. 3 and 4). We speculated that the differences in their response to MeJA observed in our study might be due to the different number of MeJA-responsive elements in the promoters of individual CaNHL genes. ROS have been reported to act as antimicrobial agents and cross-links within the plant cell wall to block pathogen entry or to serve as local and systemic secondary messengers to trigger immune responses, such as the expression of defense-related genes or stomatal closure $^{32-35}$. Xu et al. reported that UV-primed strawberry leaves induced disease resistance involving ROS production $^{36}$. Similarly, we found that CaNHL4 is able to induce the production of ROS, resulting in a defense response against pathogen infection (Fig. 9).

It has been documented that JA and SA function as endogenous growth regulators that induce systemic resistance in plants ${ }^{37}$. Peng et al. treated $N$. benthamiana leaves with exogenous MeJA and found that the expression of NbHIN1 was induced at $5 \mathrm{~h}$ after treatment, suggesting that NbHIN1 may be involved in the JA pathway ${ }^{38}$. In this study, we found that the expression levels of CaNHL1, CaNHL4, CaNHL6, CaNHL10, CaNHL11, and CaNHL12 significantly increased after exogenous MeSA treatment (Fig. 5), indicating that CaNHL may also be involved in the response of pepper to SA. Furthermore, our results showed that the CaNHL1, CaNHL4, and CaNHL12 genes are highly induced in response to the infection by P. capsici, P. syringae, and TMV (Fig. 6), which further confirms that these genes might be involved in the biotic stress response of pepper. Interestingly, we found that the CaNHL genes significantly induced in response to abiotic and biotic stresses clustered into the same group (I or III) based on the phylogenetic tree analysis (Fig. 1). These findings suggested that NHL family members in group I and group III may be involved in plant defense. Therefore, we speculate that the $N H L$ genes of S. lycopersicum and N. tabacum in this subgroup may also participate in disease resistance.

Numerous studies have shown that NHL proteins in the NHL family localize to the plasma membrane ${ }^{38-40}$.
Similarly, the present localization study of CaNHL4 revealed its localization to the plasma membrane (Fig. 8). Studies on the disease resistance protein AtNDR1 have shown that the membrane-localized AtNDR1 protein tends to accumulate in the cell membrane near the infection site, which facilitates quick sensing of pathogens, thus resulting in rapid activation of the defense respons $\mathrm{e}^{39,41}$. CaNHL4 localizes to the plasma membrane, and it is speculated that CaNHL4 may also be involved in sensing pathogens and quickly inducing a defense response. Our experiments revealed that the silencing of CaNHL4 enhances the infection of P. capsici, P. syringae, and TMV, suggesting that CaNHL4 is involved in the defense response of pepper to these three pathogens (Fig. 6). A growing body of evidence has shown that NHL family proteins are involved in plant resistance against various pathogens. For example, the expression of OsHIN1 in rice increases blast resistance ${ }^{42}$, and overexpression of AtNHL3 in A. thaliana significantly improves resistance to Pseudomonas ${ }^{39}$. Taken together, these findings suggest that CaNHL4 is involved in the rapid disease resistance of plants to pathogens.

Our findings presented in this study highlight the importance of CaNHL4 in the disease resistance of pepper. To the best of our knowledge, this is the first study to identify the CaNHL genes and examine their expression profiles in a genome-wide manner in pepper. Functional analysis showed that CaNHL is involved in JA-induced and SA-induced plant stress resistance and that CaNHL4 plays an important role in pepper stress resistance. In the future, gene editing, such as CRISPR/Cas9 and proteomics technologies, will be used to further understand the role of CaNHL4 in the JA and SA signaling pathways.

\section{Materials and methods \\ Plant materials and bacterial strains}

The pepper cultivar 'Zunla-1' (Capsicum annuum L.) and tobacco ( $N$. benthamiana) were grown in the greenhouse under a $14 \mathrm{~h} / 10 \mathrm{~h}$ light/dark photoperiod, $75 \%$ relative humidity and $25^{\circ} \mathrm{C}$.

A. tumefaciens GV3101 was grown in LB media supplemented with kanamycin and Rif at $28^{\circ} \mathrm{C}$ in an orbital shaker at $200 \mathrm{rpm}$ and harvested during the log phase of growth for infiltration.

\section{Identification of CaNHL family genes}

By using the BLASTp program, we searched the amino acid sequence of the HIN1 protein in the C. annuum Zunla-1 whole genome database of the Sol Genomics Network $^{43,44}$ (https://solgenomics.net/) and selected the sequences with $E \leq 1 \mathrm{e}-10$ as candidate sequences. ProtParam tool (https://web.expasy.org/protparam/) was used to calculate the MW, pI, and chemical formula. We then used SMART (http://smart.embl-heidelberg.de/) to 
predict the domain architectures of these proteins, and those containing NHL LEA-2 conserved domains were identified as CaNHL family genes.

\section{Bioinformatic analysis}

A phylogenetic tree was constructed using the amino acid sequences translated from the coding sequences of the $\mathrm{CaNHL}$ genes together with the amino acid sequences of SINHL of S. lycopersicum and NtHIN1 of N. tabacum. Based on the neighbor-joining (NJ) method, a phylogenetic tree was constructed by the MEGA 7.0.26 program. The bootstrap value was set to 1000 , and the Poisson model was used, allowing an estimation of the confidence of the results of the phylogenetic tree.

For regulatory element analysis, we extracted $1500 \mathrm{bp}$ upstream sequences of thee above-mentioned genes from the genome. The PlantCare database (http:// bioinformatics.psb.ugent.be/webtools/plantcare/html/)

was used to predict the regulatory element components ${ }^{45}$, and TBtools was used to display the results ${ }^{46}$.

For motif identification, the multiple expectation maximization for motif elicitation (MEME) program (http://meme.nbcr.net/meme/intro.html) was used to identify the conserved motifs in the identified CaNHL proteins ${ }^{47}$. The optimization parameters were as follows: number of repetitions, any; maximum number of motifs, 15; and optimum width of each motif, between 6 and 50 residues. TBtools was used to show the motif structure $^{46}$.

\section{RNA-Seq analysis}

By analyzing the pepper transcriptomic data available in the Pepper Informatics Hub (http://pepperhub.hzau.edu. $\mathrm{cn} /)^{48}$, we determined the expression patterns of the members of the CaNHL family in different tissues, including the leaves, stems, roots, flowers, petals, stamens, and fruits, and the expression patterns under different stress treatments, including cold, heat, $\mathrm{NaCl}, \mathrm{SA}, \mathrm{ABA}$, and JA. TBtools was used to visualize the results.

\section{Plant treatment with exogenous hormones}

Pepper (C. апnиum) plants at the six-leaf stage were sprayed with $0.1 \mathrm{mmol} / \mathrm{L}$ MeJA or MeSA (Sigma-Aldrich, USA). Mock plants were sprayed with sterile water. Samples were collected at 3-h intervals for up to $12 \mathrm{~h}$, immediately frozen in liquid nitrogen and stored at $-80^{\circ} \mathrm{C}$ for RNA isolation.

\section{Pathogen inoculation and quantitative RT-PCR}

For TMV inoculation, $100 \mu \mathrm{L}$ extracts of TMV-GFPinfected leaves were applied to each leaf by rubbing, after which leaves were imaged under UV light at 2 and $4 \mathrm{~d}$ after inoculation. Each experiment was repeated three times, with at least three independent plants used each time. For P. capsici, we inoculated fresh fungal plugs for $3 \mathrm{~d}$ into fresh pepper leaves and removed the plug on the second day after inoculation. The lesion size was observed at $3 \mathrm{dpi}$. Trypan blue staining was used to visualize the lesions. For P. syringae, a fresh bacterial overnight culture was adjusted to OD600 of 0.8 with sterile water, and then injected into the pepper leaves by a syringe. The lesion sizes were monitored and measured at 2, 4, and $6 \mathrm{dpi}$.

Real-time quantitative PCR (qPCR) was carried out by a CFX Touch Real-Time PCR System (Bio-Rad, USA) and a QuantiNova SYBR Green PCR Kit (Qiagen, Germany) to determine the relative expression level of the target gene. Primer 5.0 software was used to design gene-specific primers according to the coding sequence of each gene. $U B I 3$ was used as the internal reference gene ${ }^{49}$, and the relative changes in gene transcription levels were measured by the $2^{-\Delta \Delta C T}$ method.

\section{Vector construction}

For VIGS, pTRV was used ${ }^{50}$. A $200 \mathrm{bp}$ fragment of CaNHL4 (MN961192) was selected through the VIGS tool (https://vigs.solgenomics.net/); this fragment is specific to CaNHL4 and has no mismatches with other CaNHLs. The PCR-amplified fragment was cloned into a pTRV2 vector using the restriction sites of BamHI and XhoI.

For subcellular localization, the open-reading frame of CaNHL4 was cloned into a pART27-N-mGFP vector by using the restriction sites of EcoRI and XbaI.

For transient expression of CaNHL4, we digested the CaNHL4 fragment using the restriction sites of EcoRI and $\mathrm{XbaI}$ from pART27-N-eGFP-CaNHL4 and cloned it into a pART27-N-7*Myc vector. All the primers used are provided in Supporting Information 3.

\section{Agroinfiltration and confocal microscopy}

pART27:CaNHL4-N-mGFP, pART27-N-7*Myc-CaNHL4, and pTRV2:CaNHL4 were transformed into Agrobacterium strain GV3101. Agrobacterium-mediated transient expression was performed following the methods described previously ${ }^{51}$. For VIGS, Agrobacterium strain GV3101 carrying pTRV1, pTRV2:00, or pTRV2:CaNHL4 was adjusted to an OD600 of 0.4 and mixed at a 1:1 ratio, after which the mixture was infiltrated into the leaves of $C$. annuum plants at the four-leaf stage. For localization, infiltrated $N$. benthamiana leaves were harvested at $36 \mathrm{~h}$ after infiltration, and leaf discs were visualized using LSM780 confocal laser scanning microscope equipped with a $\times 40 / 1.2$ waterimmersion objective (Zeiss, Germany). Excitation of RFP was performed at $543 \mathrm{~nm}$ with a $\mathrm{HeNe}$ laser. A 590-620 nm filter was used to capture the emissions. Excitation of GFP was performed at $488 \mathrm{~nm}$ with an Arion laser, and the emissions were captured with a 505-530 nm pass filter. Images were scanned eight times. 


\section{Diaminobenzidine (DAB) staining}

DAB (BBI Life Science, China) was used to detect ROS production at $2 \mathrm{~d}$ after inoculation, the leaves were treated with $1 \mathrm{mg} / \mathrm{mL}$ DAB solution for $6 \mathrm{~h}$, after which the leaves were decolorized in the decolorizing solution, which was composed of ethanol:acetic acid:glycerol at a ratio of 3:1:1, at $95^{\circ} \mathrm{C}$ for $15 \mathrm{~min}$.

\section{Statistical analysis}

All the experiments and data presented here involved at least three repeats. The data are presented as the means and standard deviations. The statistical analyses were performed with SPSS software (version 19.0) using Student's $t$-test.

\section{Acknowledgements}

We would like to thank Alexander Sloan (The Australian National University, Canberra, Australia) for critical reading of our manuscript; Prof. Yule Liu (Tsinghua University, Beijing, China) for providing the PSPDK661 vector; Prof. Weixing Shan (Northwest A\&F University, Shanxi, China) for providing the VIGS vector qTRV2 and the expression vectors pART27-N-eGFP, pART27-N-7*Myc, and PM-Marker-dsRFP; and Prof. Bingsheng Qiu (Institute of Microbiology, Chinese Academy of Sciences, China, Beijing, China) for providing antibodies. This work was partly supported by The National Natural Science Foundation of China (31670148, 31870147); the Fundamental Research Funds for the Central Universities (XDJK2020B064); the Science and Technology Projects of the Chongqing Company of China Tobacco Corporation (NY20180401070001, NY20180401070008, NY20180401070010); and Southwest University's Training Program of Innovation and Entrepreneurship for Undergraduates (Project No. X201910635133).

\section{Author details}

'Laboratory of plant immunity and ecological control of plant disease, College of Plant Protection, Southwest University, 400716 Chongqing, China. ${ }^{2}$ Department of Botany and Plant Biology, Section of Biology, Faculty of Science, University of Geneva, 1211 Geneva 4, Switzerland. ${ }^{3}$ State Key Laboratory of North China Crop Improvement and Regulation, College of Plant Protection, Hebei Agricultural University, 071001 Baoding, China

\section{Conflict of interest}

The authors declare that they have no conflict of interest.

Supplementary Information accompanies this paper at (https://doi.org/ 10.1038/s41438-020-0318-0).

Received: 16 December 2019 Revised: 31 March 2020 Accepted: 6 April 2020

Published online: 01 June 2020

\section{References}

1. Martin, G. B., Bogdanove, A. J. \& Sessa, G. Understanding the functions of plant disease resistance proteins. Annu. Rev. Plant Biol. 54, 23 (2003).

2. Burgh, Avd \& Joosten, M. H. A. J. Plant immunity: thinking outside and inside the box. Trends Plant Sci. 24, 587-601 (2019).

3. Gao, J.-S. et al. The TIR-NBS but not LRR domains of two novel N-like proteins are functionally competent to induce the elicitor p50-dependent hypersensitive response. Physiol. Mol. Plant Pathol. 71, 0-87 (2007).

4. Dodds, P. N. et al. Direct protein interaction underlies gene-for-gene specificity and coevolution of the flax resistance genes and flax rust avirulence genes. Proc. Natl Acad. Sci. USA 103, 8888-8893 (2006).

5. Balint-Kurti, P. The plant hypersensitive response: concepts, control and consequences. Mol. Plant Pathol. 20, 1163-1178 (2019).

6. Delledonne, M., Zeier, Jr., Marocco, A. \& Lamb, C. Signal interactions between nitric oxide and reactive oxygen intermediates in the plant hypersensitive disease resistance response. Proc. Natl Acad. Sci. USA 98, 13454-13459 (2001).

7. Century, K. S. et al. NDR1, a pathogen-induced component required for Arabidopsis disease resistance. Science 278, 1963-1965 (1997).

8. Takahashi, Y. et al. Identification of tobacco HIN1 and two closely related genes as spermine-responsive genes and their differential expression during the tobacco mosaic virus induced hypersensitive response and during leaf and flower senescence. Plant Mol. Biol. 54, 613-622 (2004).

9. Peng, H. et al. Cloning, expression and anti-virus function analysis of tomato resistance-related gene SIHin1. Sci. Agric. Sin. 7, 1242-1251 (2017).

10. Kshamata et al. LEA proteins prevent protein aggregation due to water stress. Biochem. J. 388, 151-157 (2005).

11. Maldonado, A. et al. Overexpression of four Arabidopsis thaliana NHL genes in soybean (Glycine max) roots and their effect on resistance to the soybean cyst nematode (Heterodera glycines). Physiol. Mol. Plant Pathol. 86, 1-10 (2014).

12. Chen, Q. et al. StPOTHR1, a NDR1/HIN1-like gene in Solanum tuberosum, enhances resistance against Phytophthora infestans. Biochem. Biophys. Res. Commun. 496, 1155-1161 (2018).

13. Chong, J., Henanff, G. L., Bertsch, C. \& Walter, B. Identification, expression analysis and characterization of defense and signaling genes in Vitis vinifera. Plant Physiol. Biochem. 46, 469-481 (2008).

14. Ming, S. Z. et al. Identification of the cis-acting elements in Arabidopsis thaliana NHL10 promoter responsible for leaf senescence, the hypersensitive response against Cucumber mosaic virus infection, and spermine treatment. Plant Sci. 168, 415-422 (2005).

15. Varet, A. et al. NHL25 and NHL3, two NDR1/HIN1-like genes in Arabidopsis thaliana with potential role(s) in plant defense. Mol. Plant Microbe Interact. 15, 608-616 (2002).

16. Seskar, M., Shulaev, V. \& Raskin, I. Endogenous methyl salicylate in pathogeninoculated tobacco plants. Plant Physiol. 116, 387 (1998).

17. Shulaev, V., Silverman, F. \& Raskin, I. Airborne signaling by methyl salicylate in plant pathogen resistance. Nature 385, 718-721 (1997).

18. Cheong, J.-J. \& Choi, Y. Methyl jasmonate as a vital substance in plants. Trends Genet. 19, 409-413 (2003).

19. Zhu, F. et al. Alpha-momorcharin, a RIP produced by bitter melon, enhances defense response in tobacco plants against diverse plant viruses and shows antifungal activity in vitro. Planta 237, https://doi.org/10.1007/s00425-0121746-3 (2012)

20. Yan, J. et al. The Arabidopsis coronatine insensitive 1 protein is a jasmonate receptor. Plant Cell 21, 2220-2236 (2009).

21. $L I, J . \&$ LI, C. Seventy-year major research progress in plant hormones by Chinese scholars. Sci. Sin. Vitae 49, 1227-1281 (2019).

22. Chen, W., Chao, G. \& Singh, K. The promoter of a H2O2-inducible, Arabidopsis glutathione S-transferase gene contains closely linked OBF- and OBP1binding sites. Plant J. 10, 955-966 (1997).

23. Gopalan, S., Wei, W. \& He, S. hrp gene-dependent induction of hin1: a plant gene activated rapidly by both harpins and the avrPto gene-mediated signal. Plant J. 10, 591-600 (1996).

24. Goyal, K, Walton, L. J. \& Tunnacliffe, A. LEA proteins prevent protein aggregation due to water stress. Biochem. J. 388, 151-157 (2005).

25. Zegzouti, $H$. et al. ER5, a tomato CDNA encoding an ethylene-responsive LEAlike protein: characterization and expression in response to drought, $A B A$ and wounding. Plant Mol. Biol. 35, 847-854 (1997).

26. Singh, S. et al. Solution structure of a late embryogenesis abundant protein (LEA14) from Arabidopsis thaliana, a cellular stress-related protein. Protein Sci. 14, 2601-2609 (2005)

27. Maldonado, A. et al. Overexpression of four Arabidopsis thaliana NHL genes in soybean (Glycine max) roots and their effect on resistance to the soybean cyst nematode (Heterodera glycines). Physiol. Mol. Plant Pathol. 86, 1-10, https://doi. org/10.1016/j.pmpp.2014.02.001 (2014).

28. Wang, X. M. et al. Involvement of abscisic acid and salicylic acid in signal cascade regulating bacterial endophyte-induced volatile oil biosynthesis in plantlets of Atractylodes lancea. Physiol. Plant. 153, 30-42 (2015).

29. Hildmann, T. et al. General roles of abscisic and jasmonic acids in gene activation as a result of mechanical wounding. Plant Cell 4, 1157-1170 (1992).

30. Rezzonico, E., Flury, N., Meins, F. \& Beffa, R. Transcriptional down-regulation by abscisic acid of pathogenesis-related beta-1,3-glucanase genes in tobacco cell cultures. Plant Physiol. 117, 585-592 (1998).

31. Becker, D. et al. Regulation of the ABA-sensitive Arabidopsis potassium channel gene GORK in response to water stress. FEBS Lett. 554, 119-126 (2003). 
32. Waszczak, C., Carmody, M. \& Kangasjärvi, J. Reactive oxygen species in plant signaling. Annu. Rev. Plant Biol. 69, https://doi.org/10.1146/annurev-arplant042817-040322 (2018).

33. Lamb, C. \& Dixon, R. The oxidative burst in plant disease resistance. Annu. Rev. Plant Physiol. Plant Mol. Biol. 48, 251-275 (1997).

34. Kadota, Y., Shirasu, K. \& Zipfel, C. Regulation of the NADPH oxidase RBOHD during plant immunity. Plant Cell Physiol. 56, https://doi.org/10.1093/pcp/ pcr063 (2015).

35. Suzuki, N., Koussevitzky, S., Mittler, R. \& Miller, G. ROS and redox signaling in the response of plants to abiotic stress. Plant Cell Environ. 35, 259-270 (2011).

36. $\mathrm{Xu}, \mathrm{Y}$. et al. Ultraviolet-C priming of strawberny leaves against subsequent Mycosphaerella fragariae infection involves the action of reactive oxygen species, plant hormones, and terpenes. Plant Cell Environ. 42, 815-831 (2019).

37. Pieterse, C., Leon-Reyes, A., Ent, S. \& van Wees, S. Networking by smallmolecule hormones in plant immunity. Nat. Chem. Biol. 5, 308-316 (2009).

38. Peng, $\mathrm{H}$. et al. Overexpression of a pathogenesis-related gene NbHIN1 confers resistance to Tobacco Mosaic Virus in Nicotiana benthamiana by potentially activating the jasmonic acid signaling pathway. Plant Sci. 283, 147-156 (2019).

39. Varet, A., Hause, B., Hause, G., Scheel, D. \& Lee, J. The Arabidopsis NHL3 gene encodes a plasma membrane protein and its overexpression correlates with increased resistance to Pseudomonas syringae pv. tomato DC3000. Plant Physiol. 132, 2023-2033 (2003)

40. Coppinger, P. et al. Overexpression of the plasma membrane-localized NDR1 protein results in enhanced bacterial disease resistance in Arabidopsis thaliana. Plant J. 40, 225-237 (2004).

41. Knepper, C., Savory, E. A. \& Day, B. The role of NDR1 in pathogen perception and plant defense signaling. Plant Signal. Behav. 6, 1114-1116 (2011).
42. Rakwal, R., Agrawal, G. K., Tamogami, S. \& Iwahashi, H. Transcriptional profiling of OsHin1 in rice plants: a potential role in defense/stress and development Plant Sci. 166, 997-1005 (2004).

43. Fernandez-Pozo, N. et al. The Sol Genomics Network (SGN) - from genotype to phenotype to breeding. Nucleic Acids Res. 43, D1036-D1041 (2015).

44. Qin, C. et al. Whole-genome sequencing of cultivated and wild peppers provides insights into Capsicum domestication and specialization. Proc. Nat/ Acad. Sci. USA 111, 5135 (2014).

45. Magali, L. et al. PlantCARE, a database of plant cis-acting regulatory elements and a portal to tools for in silico analysis of promoter sequences. Nucleic Acids Res. 30, 325-327 (2002).

46. Chen, C., Xia, R., Chen, H. \& He, Y. TBtools, a Toolkit for Biologists integrating various HTS-data handling tools with a user-friendly interface. Preprint at 289660, 10.1101/289660 \%J bioRxiv (2020).

47. Bailey, T. et al. MEME SUITE: tools for motif discovery and searching. Nucleic Acids Res. 37, W202-W208 (2009).

48. Liu, F. et al. PepperHub, a Pepper Informatics Hub for the chili pepper research community. Mol. Plant 10, S167420521730076X (2017).

49. Wan, Hongjian et al. Identification of reference genes for reverse transcription quantitative real-time PCR normalization in pepper (Capsicum annuum L.) Biochem. Biophys. Res. Commun. 416, 24-30 (2011).

50. Liu, Y., Schiff, M., Marathe, R. \& Kumar, D. Tobacco Rar1, EDS1 and NPR1/NIM1 like genes are required for $\mathrm{N}$-mediated resistance to tobacco mosaic virus. Plant J. 30, 415-429 (2002)

51. Ma, L., Lukasik, E., Gawehns, F. \& Takken, F. in Plant Fungal Pathogens: Methods and Protocols, Vol. 835 Methods in Molecular Biology (eds Bolton, M. D. \& Thomma, Bphj) 61-74 (Humana Press Inc., Totowa, NJ, 2012). 